\title{
ERRATUM
}

\section{Maintenance and growth requirements for nitrogen, lysine and methionine and their utilisation efficiencies in juvenile black tiger shrimp, Penaeus monodon, using a factorial approach - ERRATUM}

\author{
Lenaïg Richard, Pierre-Philippe Blanc, Vincent Rigolet, Sadasivam J. Kaushik and Inge Geurden \\ doi:10.1017/S0007114509992844, Published by Cambridge University Press, 30 Nov 2009.
}

The publishers regret to announce that there were some errors in Table 1 of this paper, namely that some superscripts were incorrect.

The correct version of the table is below. We apologise for any inconvenience caused.

Table 1. Formulation and analysed composition of the ten experimental semi-purified diets fed to Penaeus monodon juveniles for 6 weeks

\begin{tabular}{|c|c|c|c|c|c|c|c|c|c|c|}
\hline & \multicolumn{10}{|c|}{ Diets } \\
\hline & NP & LP & LPM & LPL & MP & MPM & MPL & $\mathrm{HP}$ & HPM & HPL \\
\hline \multicolumn{11}{|l|}{ Ingredients ( $\mathrm{g} / \mathrm{kg}$ diet) } \\
\hline Casein* & 0 & 62 & 62 & 62 & 186 & 186 & 186 & 310 & 310 & 310 \\
\hline Amino acid mix $†$ & 0 & 45 & 45 & 45 & 135 & 135 & 135 & 225 & 225 & 225 \\
\hline Cholesterol* & 20 & 20 & 20 & 20 & 20 & 20 & 20 & 20 & 20 & 20 \\
\hline Soybean lecithin $\ddagger$ & 20 & 20 & 20 & 20 & 20 & 20 & 20 & 20 & 20 & 20 \\
\hline Fish oil§ & 60 & 60 & 60 & 60 & 60 & 60 & 60 & 60 & 60 & 60 \\
\hline Sodium alginateł & 50 & 50 & 50 & 50 & 50 & 50 & 50 & 50 & 50 & 50 \\
\hline Mineral mix\| & 50 & 50 & 50 & 50 & 50 & 50 & 50 & 50 & 50 & 50 \\
\hline Vitamin mix & 50 & 50 & 50 & 50 & 50 & 50 & 50 & 50 & 50 & 50 \\
\hline Agar* & 15 & 15 & 15 & 15 & 15 & 15 & 15 & 15 & 15 & 15 \\
\hline Cellulose & 20 & 20 & 20 & 20 & 20 & 20 & 20 & 20 & 20 & 20 \\
\hline Fish protein soluble concentrate§ & 20 & 20 & 20 & 20 & 20 & 20 & 20 & 20 & 20 & 20 \\
\hline Gelatinised maize starch ${ }^{\star *}$ & 680 & 573 & 573 & 573 & 359 & 358 & 359 & 144 & 144 & 144 \\
\hline Attractant mix $\dagger \dagger$ & 15 & 15 & 15 & 15 & 15 & 15 & 15 & 15 & 15 & 15 \\
\hline \multicolumn{11}{|l|}{ Analysed chemical composition } \\
\hline DM (\% diet) & $89 \cdot 6$ & $89 \cdot 3$ & $90 \cdot 6$ & $90 \cdot 4$ & $90 \cdot 6$ & $89 \cdot 8$ & 89.5 & $89 \cdot 0$ & $89 \cdot 2$ & 89.1 \\
\hline $\mathrm{N}(\% \mathrm{DM})$ & 0.82 & $2 \cdot 31$ & $2 \cdot 71$ & $2 \cdot 31$ & 5.45 & 5.44 & $5 \cdot 28$ & 8.52 & 8.65 & 8.62 \\
\hline Crude protein $(\mathrm{N} \times 6.25, \% \mathrm{DM})$ & $5 \cdot 1$ & $14 \cdot 4$ & $16 \cdot 9$ & $14 \cdot 4$ & $34 \cdot 1$ & 34.0 & 33.0 & 53.2 & $54 \cdot 1$ & 53.9 \\
\hline Lys (\% DM) & 0.18 & 0.91 & 1.04 & 0.66 & 2.35 & 2.39 & 1.62 & 3.63 & 3.68 & 2.56 \\
\hline Met (\% DM) & 0.10 & 0.44 & 0.38 & 0.47 & 0.96 & 0.69 & 0.99 & 1.54 & 1.12 & 1.60 \\
\hline Crude lipid (\% DM) & $6 \cdot 8$ & $6 \cdot 8$ & $7 \cdot 0$ & $7 \cdot 4$ & $7 \cdot 8$ & 6.9 & $7 \cdot 7$ & 7.5 & 7.5 & $7 \cdot 7$ \\
\hline Ash (\% DM) & 5.7 & $5 \cdot 7$ & 5.8 & 5.7 & $6 \cdot 0$ & $6 \cdot 0$ & $6 \cdot 0$ & 5.9 & $6 \cdot 0$ & $6 \cdot 1$ \\
\hline Gross energy (kJ/g DM) & $18 \cdot 6$ & $19 \cdot 0$ & $19 \cdot 3$ & $19 \cdot 2$ & $20 \cdot 2$ & $20 \cdot 1$ & $19 \cdot 6$ & $21 \cdot 3$ & $21 \cdot 0$ & $21 \cdot 0$ \\
\hline
\end{tabular}

NP, non-protein; LP, low protein; LPM, methionine-deficient low-protein diets; LPL, lysine-deficient low-protein diets; MP, medium protein; MPM, methionine-deficientmediumprotein diets; MPL, lysine-deficient medium-protein diets; HP, high protein; HPM, methionine-deficient high-protein diets; HPL, lysine-deficient high-protein diets.

*Acros France; $95 \%$ stabilised cholesterol; $98 \%$ glycine; $98 \%$ D-glucosamine; $\mathrm{HCl}$; agar powder; pure casein (CAS 9000-71-9).

† Eurolysine and Acros (see Table 2 for details).

‡ Louis François (St Maur, France).

§Sopropêche (Lorient, France).

I Supplied the following (to provide $\mathrm{g} / \mathrm{kg}$ mixture): magnesium oxide, 124 ; calcium carbonate, $215 ; \mathrm{KCl}, 90 ; \mathrm{NaCl}, 40 ; \mathrm{KI}, 40 \mathrm{mg} ; \mathrm{copper}$ sulphate, 3 ; cobalt sulphate, $20 \mathrm{mg}$; ferric sulphate, 20; manganese sulphate, $3 ; \mathrm{ZnSO}_{4}$, 4; dibasic calcium phosphate, 500; NAF, 1.

1 Supplied the following (to provide $\mathrm{g} / \mathrm{kg}$ mixture): retinyl acetate $(A), 0.172,1$; thiamin $\left(B_{1}\right), 0.1$; riboflavin $\left(B_{2} ; 80 \%\right)$, 0.5; nicotinic acid $\left(B_{3}\right)$, $1 ;$ calcium pantothenate $\left(B_{5}, 98 \%\right), 2$; pyridoxine $\left(B_{6}\right), 0.3$; inositol $\left(B_{7}\right), 30$; biotin $\left(B_{8}, 2 \%\right), 1$; folic acid $\left(B_{9}\right), 0.1 ;$ vitamin $B_{12}(1 \mathrm{~g} / \mathrm{kg}), 1 ;$ ascorbic acid $(C, 35 \%), 14 \cdot 29 ;$ cholecalciferol $\left(D_{3}\right), 0.006$; tocopheryl acetate $(\mathrm{E}), 3 \cdot 7$; menadione $\left(\mathrm{K}_{3}, 50 \%\right)$, 2; choline chloride $(60 \%), 167$.

${ }^{\star *}$ Roquette (Lestrem, France).

t† Contained glucosamine, taurine, betaine, glycine and alanine as 5:3:3:2:2.

\section{Reference}

1. Richard L, Blanc P-P, Rigolet V, et al. (2010) Maintenance and growth requirements for nitrogen, lysine and methionine and their utilisation efficiencies in juvenile black tiger shrimp, Penaeus monodon, using a factorial approach. Br J Nutr 103, 984-995. Published by Cambridge University Press, 30 November 2009, doi:10.1017/S0007114509992844. 